\title{
Dual Learning: Theoretical Study and an Algorithmic Extension
}

\author{
${ }^{1}$ Zhibing Zhao, ${ }^{2}$ Yingce Xia, ${ }^{2}$ Tao Qin, ${ }^{1}$ Lirong Xia, ${ }^{2}$ Tie-Yan Liu \\ ${ }^{1}$ Rensselaer Polytechnic Institute ${ }^{2}$ Microsoft Research Asia \\ ${ }^{1}$ zhaozb08@gmail.com ${ }^{1}$ xial@cs.rpi.edu ${ }^{2}$ \{yingce.xia, taoqin,tyliu\}@microsoft.com
}

\begin{abstract}
Dual learning has been successfully applied in many machine learning applications including machine translation, image-to-image transformation, etc. The high-level idea of dual learning is very intuitive: if we map an $x$ from one domain to another and then map it back, we should recover the original $x$. Although its effectiveness has been empirically verified, theoretical understanding of dual learning is still very limited. In this paper, we aim at understanding why and when dual learning works. Based on our theoretical analysis, we further extend dual learning by introducing more related mappings and propose multi-step dual learning, in which we leverage feedback signals from additional domains to improve the qualities of the mappings. We prove that multi-step dual learning can boost the performance of standard dual learning under mild conditions. Experiments on WMT 14 English $\leftrightarrow$ German and MultiUN English $\leftrightarrow$ French translations verify our theoretical findings on dual learning, and the results on the translations among English, French, and Spanish of MultiUN demonstrate the effectiveness of multi-step dual learning.
\end{abstract}

\section{INTRODUCTION}

Most machine learning tasks can be formulated as learning a mapping from one domain to another one, like image classification (from image to label), neural machine translation (from the source language to the target language), speech recognition (from voice to text), etc. Among them, many tasks are of dual forms, like image classification v.s. image generation (from label to image), the neural machine translation between two languages (e.g., English $\rightarrow$ French v.s. French $\rightarrow$ English), speech recognition v.s. speech synthesis (from text to voice), etc. Such duality can be utilized to improve the model qualities.

One prominent framework is dual learning, first proposed by He et al. [2016] for machine translation and then applied to many other applications like image translation [Kim et al., 2017, Zhu et al., 2017], question answering and generation [Tang et al. 2017], etc. In dual learning, two mapping functions between two domains are trained simultaneously so that one function is close to the inverse of the other. The intuition is that, if we translate a sentence from English to French and then translate the obtained French sentence back to English, we should get the same sentence or a very similar one. Dual learning is of great interest because it can accommodate any unidirectional architecture, e.g. a transformer [Vaswani] et al. 2017], and provide a performance boost. Moreover, dual learning can be used in semi-supervised learning, which is highly desirable since deep neural networks are generally thirst for labeled data.

Despite the empirical success of dual learning, theoretical understanding is very limited. In this paper, we conduct both theoretical analyses and empirical studies to answer the following questions:

- Why and when does dual learning improve a mapping function?

- Can we further improve the performance of a mapping function?

\subsection{OUR CONTRIBUTIONS}

Our contributions are in two folds: a theoretical study of dual learning and the framework of multi-step dual learning, which subsumes dual learning as a special case. Without loss of generality, we take machine translation as an example for the study and algorithm presentation. 
Dual learning theory. We take a novel statistical approach to model the problem. Suppose there are two vanilla translators between two language spaces, one forward and the other backward. Based on our Theorem 1 . dual learning outperforms both vanilla translators under natural assumptions. Empirical studies show that an improvement is observed even if the reconstruction is far from perfect.

Multi-step dual learning. We propose the multi-step dual learning framework by extending dual learning. This framework uses dual learning as the basic building block and leverages a third, a fourth, or more languages to help boost the translator qualities between the original two languages. We prove that under mild conditions, this framework outperforms dual learning (Theorem 2). Our experiments on MultiUN dataset show a significant improvement (1.45 BLEU points, see Table 6 from dual learning.

\subsection{RELATED WORK}

Dual learning was first proposed by He et al. [2016] in the context of machine translation, where the two dual translators are updated in a reinforcement learning manner with the reconstructed distortion as the feedback signal. A similar approach proposed by Cheng et al. [2016] has the same high-level idea but their implementation is very different. Since then dual learning architectures have been proposed for other applications including image processing [Kim et al., 2017, Zhu et al. 2017], sentiment analysis [Xia et al., 2017a], image segmentation [Luo et al. 2017], etc.

Built upon the dual learning framework, Xia et al. [2017b] and Wang et al. [2018] considered the joint distribution constraint, which says the joint distribution of samples over two domains is invariant when computing from either domain. We relax this constraint for simplicity of analysis. Xia et al. [2018] proposed model-level dual learning, which shares components between the primary direction and the dual direction. Dual learning was also leveraged for unsupervised learning [Lample et al., 2018, Artetxe et al. 2018.

Despite the vast number of works related to dual learning, theoretical analysis is very limited. [Xia et al. 2017a|b] [15,16] conducted simple analysis of generalization ability in the supervised setting, which are different from our semi-supervised setting. Galanti et al. [2018] claim that dual learning does not circumvent the alignment problem, where a sentence is translated wrong by the forward translator but translated back to it by the backward translator. We show that the alignment problem occurs with a small probability under dual learning, and this probability can be further reduced by our multistep dual learning. Furthermore, their hypothesis that the translator should not be too complex is not verified in the context of machine translation.

Another line of research is back-translation [Sennrich et al. 2016a, Poncelas et al., 2018, Edunov et al., 2018], which leverages a backward translator to generate parallel data. There are two major differences between dual learning and back-translation: (1) Dual learning aims at improving the performances of all candidate models, while back-translation focuses on using a reversed model (fixed) to boost the primal model; (2) Back-translation generate synthesis offline, which are fed into the primal model; dual learning generates data iteratively, by which the quality of synthesis data is better due to the optimization of each model. Furthermore, our multi-step dual learning utilizes three or more language domains to enhance translators.

\section{PRELIMINARIES}

Let $S_{1}, \ldots, S_{k}$ be $k$ language spaces, composed of sentences in each language. For any $S_{i}$, we denote the distribution of sentences in $S_{i}$ by $\mu^{(i)}$ and let $X^{(i)}$ be the random variable, i.e. $\operatorname{Pr}\left(X^{(i)}=x\right)=\mu^{(i)}(x)$. As there are multiple sentences for the same meaning in each language, we assume there are a finite number of clusters in each language space.

Table 1: Notations

\begin{tabular}{cl}
\hline$k$ & number of language spaces \\
$S_{i}$ & $i$-th language space \\
$\mu^{(i)}$ & distribution of sentences in $S_{i}$ \\
$X^{(i)}$ & the random variable that follows $\mu^{(i)}$ \\
$x^{(i)}$ & one sample (sentence) in $S_{i}$ \\
$T_{i j}^{*}$ & the oracle translator from $S_{i}$ to $S_{j}$ \\
$T_{i j}$ & vanilla translator that translates from $S_{i}$ to $S_{j}$ \\
$p_{i j}$ & accuracy of the $T_{i j}$ \\
$T_{i j}^{d}$ & translator that translates from $S_{i}$ to $S_{j}$ \\
& trained using dual learning \\
$p_{i j}^{d}$ & accuracy of $T^{d} i j$ \\
$T_{i j}^{m}$ & translator that translates from $S_{i}$ to $S_{j}$ \\
& trained using multi-step dual learning \\
$p_{i j}^{m}$ & accuracy of $T_{i j}^{m}$ \\
\hline
\end{tabular}

Let $T_{i j}^{*}$ denote the oracle translator that maps a cluster or a sentence in the cluster from $S_{i}$ to the correct cluster of $S_{j}$. See Figure 1 for an example. There are clusters $C_{1}, C_{2}$, etc. in $S_{1}$, and an oracle translator $T_{12}^{*}$ maps any cluster (e.g., $C_{1}$ ) or an element in the cluster (e.g., $\left.x \in C_{1}\right)$ to the correct cluster $\left(T_{12}^{*}\left(C_{1}\right)\right)$, which is a set of sentences. Let $C\left(x^{(i)}\right)$ denote the cluster to which $x^{(i)}$ 

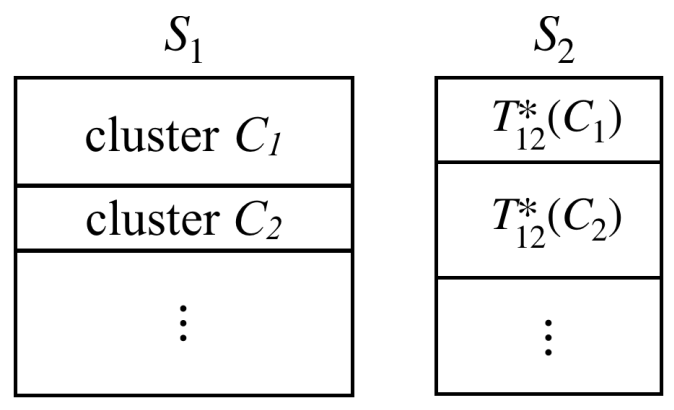

Figure 1: Illustration of two language spaces and an oracle translator.

belongs. Let $T_{i j}$ denote a vanilla translator that translates from a sentence in $S_{i}$ to one in $S_{j}$. The desired mapping is $T_{i j}\left(x^{(i)}\right) \in T_{i j}^{*}\left(x^{(i)}\right)$, where $T_{i j}^{*}$ is the oracle translator. When a sentence $x^{(i)}$ is randomly sampled from $S_{i}$ according to $\mu^{(i)}$, it is possible that this sentence is translated incorrectly. We use $p_{i j}$, the accuracy of the translator, to describe the probability of translating a sentence correctly when this sentence is randomly sampled from $S_{i}$ according to $\mu^{(i)}$. Formally,

$$
\begin{aligned}
p_{i j} & =\operatorname{Pr}_{X^{(i)} \sim \mu^{(i)}}\left(T_{i j}\left(X^{(i)}\right) \in T_{i j}^{*}\left(X^{(i)}\right)\right) \\
& =\sum_{x^{(i)} \in S_{i}, T_{i j}\left(x^{(i)}\right) \in T_{i j}^{*}\left(x^{(i)}\right)} \mu^{(i)}\left(x^{(i)}\right) .
\end{aligned}
$$

We sometimes omit the subscript $X^{(i)} \sim \mu^{(i)}$ for simplicity. It is also easy to see that $\operatorname{Pr}\left(T_{i j}\left(x^{(i)}\right) \notin T_{i j}^{*}\left(x^{(i)}\right)\right)=$ $1-p_{i j}$. In order to characterize reconstruction accuracy, we let $X^{(j, r)}$ denote the random variable that follows the distribution $T_{i j}\left(X^{(i)}\right)$ where $X^{(i)} \sim \mu^{(i)}$. We define $\mu^{(j, r)}(x)=\operatorname{Pr}\left(X^{(j, r)}=x\right)$, and further define

$$
\begin{aligned}
p_{j i}^{r} & =\operatorname{Pr}_{X^{(j, r)} \sim \mu^{(j, r)}}\left(T_{j i}\left(X^{(j, r)}\right) \in T_{j i}^{*}\left(X^{(j, r)}\right)\right) \\
& =\sum_{x^{(j, r)} \in S_{j}, T_{j i}\left(x^{(j, r)}\right) \in T_{j i}^{*}\left(x^{(j, r)}\right)} \mu^{(j, r)}\left(x^{(j, r)}\right) .
\end{aligned}
$$

The superscript $r$ means "reconstruction". The difference between $p_{j i}^{r}$ and $p_{j i}$ lies in the distributions of samples in space $S_{j}$. See Table 1 for the list of notations.

\section{THEORETICAL STUDY OF DUAL LEARNING}

We consider a semi-supervised learning task where some parallel sentences are available to train the vanilla translators in both directions, and a large amount of monolingual sentences in addition to the parallel data are available for dual learning. The structures of the translators, the way to define losses, and the optimization algorithms are decided by the designer (see Algorithm 1). W.l.o.g. we $\overline{\text { Algorithm 1: Semi-supervised Dual Learning Frame- }}$ work

Require: Parallel data $D_{12}$ for languages $S_{1}$ and $S_{2}$, additional monolingual data $D_{1}$ for $S_{1}$ and $D_{2}$ for $S_{2}$.

1: Train vanilla translators for directions $S_{1} \rightarrow S_{2}$ and $S_{2} \rightarrow S_{1}$ respectively using parallel data. The obtained vanilla translators are $T_{12}$ and $T_{21}$.

2: Continue training both translators so that the translation loss on the parallel data $D_{12}$ and reconstruction loss on monolingual datasets $D_{1}$ and $D_{2}$ are minimized. The obtained translators are $T_{12}^{d}$ and $T_{21}^{d}$.

focus on two language spaces $S_{1}$ and $S_{2}$. Recall that $T_{i j}$ denotes the vanilla translator from $S_{i}$ to $S_{j}$. For each sentence $x^{(1)} \in S_{1}$, we will focus on the 4-tuple $\left(x^{(1)}, \mu^{(1)}\left(x^{(1)}\right), T_{12}\left(x^{(1)}\right), T_{21}\left(T_{12}\left(x^{(1)}\right)\right)\right)$. Define random variables $Y_{12}$ and $Y_{21}$, which indicate whether $T_{12}$ and $T_{21}$ produce correct translations in each 4-tuple. Formally, we have

$$
Y_{12}=\left\{\begin{array}{l}
1, \text { if } T_{12}\left(x^{(1)}\right) \in T_{12}^{*}\left(x^{(1)}\right) \\
0, \text { otherwise. }
\end{array}\right.
$$

and

$$
Y_{21}=\left\{\begin{array}{l}
1, \text { if } T_{21}\left(T_{12}\left(x^{(1)}\right)\right) \in T_{21}^{*}\left(T_{12}\left(x^{(1)}\right)\right) \\
0, \text { otherwise. }
\end{array}\right.
$$

Then by definition, we have

$$
\begin{aligned}
& p_{12}=\operatorname{Pr}\left(Y_{12}=1\right) \\
& p_{21}^{r}=\operatorname{Pr}\left(Y_{21}=1\right)
\end{aligned}
$$

In order to analyze dual learning, we consider the joint distribution of $Y_{12}$ and $Y_{21}$. We use $\lambda$ to model the dependence of $Y_{12}$ and $Y_{21}$. Formally,

$$
\operatorname{Pr}\left(Y_{12}=1, Y_{21}=1\right)=p_{12} p_{21}^{r}+\lambda
$$

It's easy to see that

$$
\begin{aligned}
& \operatorname{Pr}\left(Y_{12}=1, Y_{21}=0\right)=p_{12}\left(1-p_{21}^{r}\right)-\lambda \\
& \operatorname{Pr}\left(Y_{12}=0, Y_{21}=1\right)=\left(1-p_{12}\right) p_{21}^{r}-\lambda \\
& \operatorname{Pr}\left(Y_{12}=0, Y_{21}=0\right)=\left(1-p_{12}\right)\left(1-p_{21}^{r}\right)+\lambda
\end{aligned}
$$

using (1) and (2).

Because all these probabilities are nonnegative, we have

$-\min \left\{p_{12} p_{21}^{r},\left(1-p_{12}\right)\left(1-p_{21}^{r}\right)\right\} \leq \lambda \leq \min \left\{p_{12}, p_{21}^{r}\right\}$ 
This range of $\lambda$ is not tight, but is sufficient for our analysis. The probability of the alignment issue, which means for some $x^{(1)} \in S_{1}, T_{21}\left(T_{12}\left(x^{(1)}\right)\right) \in C\left(x^{(1)}\right)$ and $Y_{12}=Y_{21}=0$, is part of $\operatorname{Pr}\left(Y_{12}=0, Y_{21}=0\right)$. We use $\delta$ to model how likely this issue occurs. Formally,

$$
p_{\text {align }}=\delta\left(\left(1-p_{12}\right)\left(1-p_{21}^{r}\right)+\lambda\right),
$$

where $0 \leq \delta \leq 1$. For translators $T_{12}^{d}$ and $T_{21}^{d}$ obtained from dual learning, we construct 4-tuples in the same way, i.e., $\left(x^{(1)}, \mu^{(1)}\left(x^{(1)}\right), T_{12}^{d}\left(x^{(1)}\right), T_{21}^{d}\left(T_{12}^{d}\left(x^{(1)}\right)\right)\right)$ and define random variables $Y_{12}^{d}$ and $Y_{21}^{d}$ similarly. Let

$$
Y_{12}^{d}=\left\{\begin{array}{l}
1, \text { if } T_{12}^{d}\left(x^{(1)}\right) \in T_{12}^{*}\left(x^{(1)}\right) \\
0, \text { otherwise. }
\end{array}\right.
$$

and

$$
Y_{21}^{d}=\left\{\begin{array}{l}
1, \text { if } T_{21}^{d}\left(T_{12}^{d}\left(x^{(1)}\right)\right) \in T_{21}^{*}\left(T_{12}^{d}\left(x^{(1)}\right)\right) \\
0, \text { otherwise. }
\end{array} .\right.
$$

We are interested in the accuracy of $T_{12}^{d}, p_{12}^{d}=\operatorname{Pr}\left(Y_{12}^{d}=\right.$ $1)$. To bridge the vanilla translators and dual translators, we make an assumption, which says if a sample in $S_{1}$ is successfully reconstructed by vanilla translators, it is also successfully reconstructed by dual translators, formally stated as follows.

Assumption 1. For any $x \in S_{1}$, if $T_{21}\left(T_{12}(x)\right) \in C(x)$, then $T_{21}^{d}\left(T_{12}^{d}(x)\right) \in C(x)$ holds.

For simplicity, we denote this case as Case 1 and the remaining cases as Case 2. Formally, for any $x \in S_{1}$,

Case 1: $T_{21}\left(T_{12}(x)\right) \in C(x)$;

Case 2: $T_{21}\left(T_{12}(x)\right) \notin C(x)$.

For any given $x \in S_{1}$ which falls in Case 2, we define

$\alpha=\operatorname{Pr}\left(T_{12}^{d}(x) \in T_{12}^{*}(x), T_{21}^{d}\left(T_{12}^{d}(x)\right) \in C(x) \mid\right.$ Case 2$)$

$\beta=\operatorname{Pr}\left(T_{12}^{d}(x) \notin T_{12}^{*}(x), T_{21}^{d}\left(T_{12}^{d}(x)\right) \in C(x) \mid\right.$ Case 2)

$\gamma=\operatorname{Pr}\left(T_{21}^{d}\left(T_{12}^{d}(x)\right) \notin C(x) \mid\right.$ Case 2$)$,

where "Case 2" denotes the condition $T_{21}\left(T_{12}(x)\right) \notin$ $C(x)$. Here $\alpha$ can be viewed as the probability of correcting the wrong translations by dual learning, $\beta$ the probability of the occurrence of the alignment problem under Case 2 , and $\gamma$ the probability of nonzero reconstruction error. $\gamma$ models the imperfectness of dual learning, which should be zero in the ideal case. It is easy to see $\alpha+\beta+\gamma=1$. The following theorem give a theoretical study of why dual learning outperforms the baseline translator by the following theorem.

Theorem 1. Under Assumption 1, for any language spaces $S_{1}$ and $S_{2}$, the accuracy of dual learning outcome $T_{12}^{d}$ is $p_{12}^{d}=(1-\alpha)\left(p_{12} p_{21}^{r}+\lambda\right)+\alpha \delta\left(p_{12}+p_{21}^{r}-\right.$ $\left.p_{12} p_{21}^{r}-\lambda\right)+\alpha(1-\delta)$, where $\lambda, \delta, \alpha$ are defined in (3), (5) and (6).
Proof. Consider a random sample $x$ and the translation from $x \in S_{1}$ to $S_{2}$. Before dual learning, the accuracy is $p_{12}$. We analyze the two cases defined earlier in Section 3

Case 1. $T_{21}\left(T_{12}(x)\right) \in C(x)$. Case 1 consists of two subcases:

Case 1.1: $T_{12}(x) \in T_{12}^{*}(x)$;

Case 1.2: $T_{12}(x) \notin T_{12}^{*}(x)$.

Although Case 1.2 is not desired, dual learning does not detect it. From (3) and (5), the probabilities of the Case 1.1 and Case 1.2 are

$$
\begin{gathered}
\operatorname{Pr}(\text { Case 1.1 })=\operatorname{Pr}\left(Y_{12}=Y_{21}=1\right)=p_{12} p_{21}^{r}+\lambda, \\
\operatorname{Pr}(\text { Case 1.2 })=p_{\text {align }}=\delta\left(\left(1-p_{12}\right)\left(1-p_{21}^{r}\right)+\lambda\right) .
\end{gathered}
$$

Case 2. $T_{21}\left(T_{12}(x)\right) \notin C(x)$. Dual learning will train the translators so that this case is minimized. The probability of this case is simply the complement of Case 1:

$$
\begin{aligned}
\operatorname{Pr}(\text { Case } 2) & =1-\left(p_{12} p_{21}^{r}+\lambda\right)-\delta\left(\left(1-p_{12}\right)\left(1-p_{21}^{r}\right)+\lambda\right) \\
& =1-\delta-(1+\delta)\left(p_{12} p_{21}^{r}+\lambda\right)+\delta\left(p_{12}+p_{21}^{r}\right) .
\end{aligned}
$$

After dual learning, Case 2 is redistributed to Case 1.1 and Case 1.2, with probabilities $\alpha$ and $\beta$ respectively. So we have

$$
\begin{aligned}
& \operatorname{Pr}\left(T_{12}^{d}(x) \in T_{12}^{*}(x), T_{21}^{d}\left(T_{12}^{d}(x)\right) \in C(x)\right) \\
= & p_{12} p_{21}^{r}+\lambda+\alpha \operatorname{Pr}(\text { Case } 2) \\
= & (1-\alpha)\left(p_{12} p_{21}^{r}+\lambda\right) \\
& +\alpha \delta\left(p_{12}+p_{21}^{r}-p_{12} p_{21}^{r}-\lambda\right)+\alpha(1-\delta),
\end{aligned}
$$

which is the accuracy of dual learning.

Relation to the vanilla translators. Observing that $1-$ $\alpha \geq 0, p_{12}+p_{21}^{r}-p_{12} p_{21}^{r}-\lambda \geq 0$ (due to (4)) and $1-\delta \geq$ 0 , the accuracy is dual learning improvement is positively correlated to the vanilla translators of both directions. The larger the $p_{12}$ or $p_{21}^{r}$ is, the higher accuracy of $T_{12}^{d}$ dual learning can achieve.

The role of $\alpha$ and $\delta$. We have $p_{12}^{d}=\alpha(1-\delta-$ $\left.(1+\delta)\left(p_{12} p_{21}^{r}+\lambda\right)+\delta\left(p_{12}+p_{21}^{r}\right)\right)+p_{12} p_{21}^{r}+\lambda$ by reorganization. So a larger $\alpha$ is desirable, which is intuitively true. Also, $p_{12}^{d}$ can be reorganized as $-\alpha \delta\left(\left(1-p_{12}\right)\left(1-p_{21}^{r}\right)+\lambda\right)+\alpha+(1-\alpha)\left(p_{12} p_{21}^{r}+\lambda\right)$, which means a small $\delta$ is desirable.

A hypothesis on $\alpha$ and $\beta$. We consider the case where the probabilities of redistribution to $\alpha$ case and $\beta$ case are proportional to $\operatorname{Pr}($ Case 1.1) and $\operatorname{Pr}($ Case 1.2). For- 
mally,

$$
\begin{aligned}
\frac{\alpha}{\beta} & =\frac{\operatorname{Pr}\left(T_{12}(x) \in T_{12}^{*}(x), T_{21}\left(T_{12}(x)\right) \in C(x)\right)}{\operatorname{Pr}\left(T_{12}(x) \notin T_{12}^{*}(x), T_{21}\left(T_{12}(x)\right) \in C(x)\right)} \\
& =\frac{p_{12} p_{21}^{r}+\lambda}{\delta\left(\left(1-p_{12}\right)\left(1-p_{21}^{r}\right)+\lambda\right)} .
\end{aligned}
$$

Then we have

$$
\begin{aligned}
p_{12}^{d} & =\frac{\left(p_{12} p_{21}^{r}+\lambda\right)\left(1-\gamma\left(1-p_{12} p_{21}^{r}-\lambda-\delta\left(\left(1-p_{12}\right)\left(1-p_{21}^{r}\right)+\lambda\right)\right)\right)}{p_{12} p_{21}^{r}+\lambda+\delta\left(\left(1-p_{12}\right)\left(1-p_{21}^{r}\right)+\lambda\right)} \\
& =\frac{\left(p_{12} p_{21}^{r}+\lambda\right)(1-\Gamma)}{p_{12} p_{21}^{r}+\lambda+\delta\left(\left(1-p_{12}\right)\left(1-p_{21}^{r}\right)+\lambda\right)},
\end{aligned}
$$

where $\Gamma=\gamma\left(1-p_{12} p_{21}^{r}-\lambda-\delta\left(\left(1-p_{12}\right)\left(1-p_{21}^{r}\right)+\lambda\right)\right)$. To compare $p_{12}^{d}$ with the accuracy of the original translator, we compute the difference

$$
\begin{aligned}
& p_{12}^{d}-p_{12} \\
= & p_{12}\left(\frac{\left(p_{21}^{r}+\lambda / p_{12}\right)(1-\Gamma)}{p_{12} p_{21}^{r}+\lambda++\delta\left(\left(1-p_{12}\right)\left(1-p_{21}^{r}\right)+\lambda\right)}-1\right) \\
= & p_{12}\left(\frac{p_{21}^{r}+\lambda / p_{12}}{p_{12} p_{21}^{r}+\lambda+\delta\left(\left(1-p_{12}\right)\left(1-p_{21}^{r}\right)+\lambda\right)}-1-\Gamma \Delta\right) \\
= & p_{12}\left(\frac{\left((1+\delta) p_{21}^{r}-\delta\right)\left(1-p_{12}\right)+\lambda\left(1 / p_{12}-1+\delta\right)}{p_{12} p_{21}^{r}+\lambda+\delta\left(\left(1-p_{12}\right)\left(1-p_{21}^{r}\right)+\lambda\right)}-\Gamma \Delta\right)
\end{aligned}
$$

where $\Delta=\frac{p_{21}^{r}+\lambda / p_{12}}{p_{12} p_{21}^{r}+\lambda+\delta\left(\left(1-p_{12}\right)\left(1-p_{21}^{r}\right)+\lambda\right)}$. Ideally, we have $\gamma=0$, which means $\Gamma=0$. If $p_{21}^{r}>\frac{\delta}{1+\delta}$, the outcome of dual learning is better than the vanilla translator. This condition is very mild because $\delta$ is small in general. The expression with the $\Gamma$ factor is negative, which is consistent with the intuition that $\gamma$ should be minimized.

\section{EXTENSION: MULTI-STEP DUAL LEARNING}

In Theorem 1, we found that both $p_{i j}$ and $p_{j i}^{r}$ play positive roles in improving $p_{i j}^{d}$ under mild assumptions. A natural question is whether this probability could be further enhanced by exploiting multiple language domains. Therefore, we propose the frameworks of multi-step dual learning, leveraging multiple languages and significantly extend the standard dual learning.

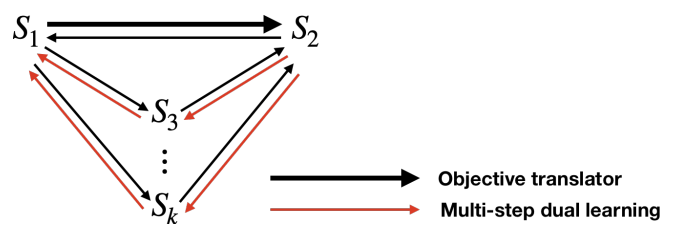

Figure 2: The proposed multi-step dual learning framework.

The proposed frameworks are illustrated in Figure 2, Let $S_{1}$ and $S_{2}$ denote the source language space and the target language space respectively. To use these frameworks, we first train the following translators: $S_{1} \leftrightarrow S_{2}$, $S_{1} \leftrightarrow S_{k}$ and $S_{2} \leftrightarrow S_{k}$ where $k \geq 3$. Then, we require a sentence from $S_{2}$ to be very similar to $S_{2} \rightarrow$ $S_{k} \rightarrow S_{1} \rightarrow S_{2}$ (or equivalently, a sentence from $S_{1}$ to be very similar to $S_{1} \rightarrow S_{2} \rightarrow S_{k} \rightarrow S_{1}$ ); In this way, we build another constraint, where the translation $S_{1} \rightarrow S_{2}$ could leverage the information pivoted by the domain $S_{k}$. In practice, to use multi-step dual learning to enhance the $S_{1} \rightarrow S_{2}$ model, we need to minimize $\sum_{x^{(2)} \in S_{2}} D\left(T_{12}\left(T_{k 1}\left(T_{2 k}\left(x^{(2)}\right)\right)\right), x^{(2)}\right)$, where $D(\cdot, \cdot)$ measures the differences of two inputs. Similar update could also be applied to $S_{2} \rightarrow S_{1}$ translation and leverage more language domains. If no auxiliary domain is provided, multi-step dual learning will degenerate to the standard dual learning. We design sampling based algorithms for this framework. Let $\theta_{i j}$ denote the parameters of translator $T_{i j}$. The algorithm is formally shown as Algorithm 2,

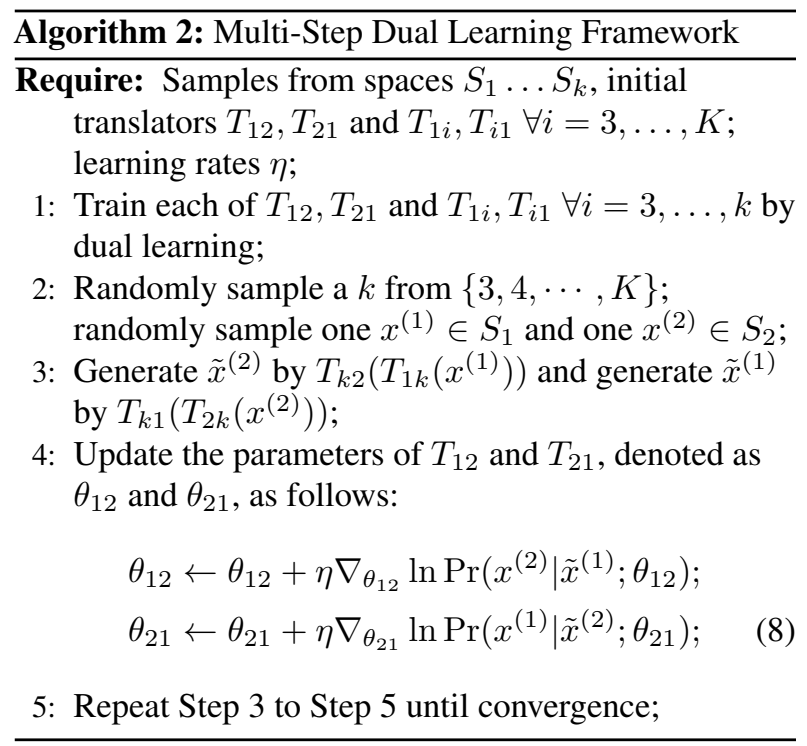

\subsection{THEORETICAL ANALYSIS}

We provide a theoretical analysis of this framework. For simplicity, we focus on the triangle structure that contains only $S_{1}, S_{2}$ and $S_{3}$. For each sentence $x \in S_{1}$, we will focus on the 5-tuple $\left(x, \mu^{(1)}(x), T_{12}^{d}(x), T_{23}^{d}\left(T_{12}^{d}(x)\right), T_{31}^{d}\left(T_{23}^{d}\left(T_{12}^{d}(x)\right)\right)\right)$.

Define random variables $Z_{12}, Z_{23}$ and $Z_{31}$, which indicate whether $T_{12}^{d}, T_{23}^{d}$ and $T_{31}^{d}$ produce correct translations in each 5-tuple. Formally, we have

$$
Z_{12}=\left\{\begin{array}{l}
1, \text { if } T_{12}^{d}(x) \in T_{12}^{*}(x) \\
0, \text { otherwise }
\end{array}\right.
$$




$$
Z_{23}=\left\{\begin{array}{l}
1, \text { if } T_{23}^{d}\left(T_{12}^{d}(x)\right) \in T_{23}^{*}\left(T_{12}^{d}(x)\right) \\
0, \text { otherwise }
\end{array}\right.
$$

and

$$
Z_{31}=\left\{\begin{array}{l}
1, \text { if } T_{31}^{d}\left(T_{23}^{d}\left(T_{12}^{d}(x)\right)\right) \in T_{31}^{*}\left(T_{23}^{d}\left(T_{12}^{d}(x)\right)\right) \\
0, \text { otherwise. }
\end{array}\right.
$$

We define $q_{12}=\operatorname{Pr}\left(Z_{12}=1\right), q_{23}=\operatorname{Pr}\left(Z_{23}=1\right)$, and $q_{31}=\operatorname{Pr}\left(Z_{31}=1\right)$. For simplicity, we assume the same dependence on any two of $Z_{12}, Z_{23}, Z_{31}$. Formally,

$$
\begin{aligned}
& \operatorname{Pr}\left(Z_{12}=Z_{23}=1\right)=q_{12} q_{23}+\lambda_{1} \\
& \operatorname{Pr}\left(Z_{23}=Z_{31}=1\right)=q_{23} q_{31}+\lambda_{1} \\
& \operatorname{Pr}\left(Z_{12}=Z_{31}=1\right)=q_{12} q_{31}+\lambda_{1}
\end{aligned}
$$

We let

$$
\operatorname{Pr}\left(Z_{12}=Z_{23}=Z_{31}=1\right)=q_{12} q_{23} q_{31}+\lambda_{2},
$$

where $\lambda_{2}$ captures the dependence of all three variables. Then the joint distribution of $Z_{12}, Z_{23}$, and $Z_{31}$ can be written as expressions of $\lambda_{1}$ and $\lambda_{2}$. Similar to the analysis for dual learning, we use $\delta$ to describe how likely $T_{31}^{d}\left(T_{23}^{d}\left(T_{12}^{d}(x)\right)\right) \in C(x)$ occurs when one or more of the three translators give incorrect translations. Formally, for any $x \in S_{1}$ and $Z_{12}, Z_{23}, Z_{31}$ s.t. $Z_{12}+Z_{23}+Z_{31} \leq 2, \operatorname{Pr}\left(T_{31}^{d}\left(T_{23}^{d}\left(T_{12}^{d}(x)\right)\right) \in\right.$ $\left.C(x) \mid Z_{12}, Z_{23}, Z_{31}\right)=\delta \operatorname{Pr}\left(Z_{12}, Z_{23}, Z_{31}\right)$. Now we are interested in the accuracy of $T_{12}^{m}$ obtained from multistep dual learning, which is $q_{12}^{m}=\operatorname{Pr}\left(Z_{12}=1\right)$. To bridge $q_{i j}$ and $q_{i j}^{m}$, we make the following assumption, which says if a sentence is successfully reconstructed in a cycle by translators obtained from dual learning, it will also be successfully reconstructed in a cycle by translators obtained from multi-step dual learning.

Assumption 2. For any $x \in S_{1}$, if $T_{31}^{d}\left(T_{23}^{d}\left(T_{12}^{d}(x)\right)\right) \in$ $C(x)$, then $T_{31}^{m}\left(T_{23}^{m}\left(T_{12}^{m}(x)\right)\right) \in C(x)$.

For simplicity, we denote the invariant case as Case 1 and the remaining cases as Case 2. Formally, for any $x \in S_{1}$,

We focus on the following two cases:

Case 1: $T_{31}^{d}\left(T_{23}^{d}\left(T_{12}^{d}(x)\right)\right) \in C(x)$;

Case 2: $T_{31}^{d}\left(T_{23}^{d}\left(T_{12}^{d}(x)\right)\right) \notin C(x)$.

Multi-step dual learning will train the translators so that Case 2 is minimized. To quantify this effect, we define the following probabilities:

$$
\begin{aligned}
& \alpha^{\prime}=\operatorname{Pr}\left(T_{12}^{m}(x) \in T_{12}^{*}(x), T_{31}^{m}\left(T_{23}^{m}\left(T_{12}^{m}(x)\right)\right) \in C(x) \mid\right. \text { Case 2) } \\
& \beta^{\prime}=\operatorname{Pr}\left(T_{12}^{m}(x) \notin T_{12}^{*}(x), T_{31}^{m}\left(T_{23}^{m}\left(T_{12}^{m}(x)\right)\right) \in C(x) \mid\right. \text { Case 2) } \\
& \gamma^{\prime}=\operatorname{Pr}\left(T_{31}^{m}\left(T_{23}^{m}\left(T_{12}^{m}(x)\right)\right) \notin C(x) \mid\right. \text { Case 2), }
\end{aligned}
$$

where Case 2 denotes the condition $T_{31}^{d}\left(T_{23}^{d}\left(T_{12}^{d}(x)\right)\right) \notin$ $C(x) . \quad \alpha^{\prime}, \beta^{\prime}, \gamma^{\prime}$ can be viewed as the probability of correcting the wrong translations by multi-step dual learning, the probability of the occurrence of the alignment problem under Case 2, and the probability of nonzero reconstruction error. $\gamma^{\prime}$ models the imperfectness of dual learning. And we have $\alpha^{\prime}+\beta^{\prime}+\gamma^{\prime}=1$.

We have the following theorem about the triangle structure. The more general case can be viewed as adding one path a time so Theorem 2 can be applied.

Theorem 2. Given languages spaces $S_{1}, S_{2}$, and $S_{3}$, where the objective is to train a translator that maps from $S_{1}$ to $S_{2}$ and under Assumption 2 the accuracy of multi-step dual learning outcome $q_{12}^{m}$ is

$$
\begin{aligned}
& \left(1-\alpha^{\prime}\right)\left(q_{12} q_{23} q_{31}+\delta q_{12}\left(1-q_{23}\right)\left(1-q_{31}\right)+(1+\delta) \lambda_{2}\right) \\
& +\alpha^{\prime}\left(1-\delta\left(1-q_{12}\right)\left(1-q_{23} q_{31}-\lambda_{1}+\lambda_{2}\right)\right.
\end{aligned}
$$

Proof. We focus on the mapping from $x \in S_{1}$ to $S_{2}$ and consider the following two cases:

Case 1: $T_{31}^{d}\left(T_{23}^{d}\left(T_{12}^{d}(x)\right)\right) \in C(x)$;

Case 2: $T_{31}^{d}\left(T_{23}^{d}\left(T_{12}^{d}(x)\right)\right) \notin C(x)$.

Case 1: Recall the definitions of $Z_{12}, Z_{23}$ and $Z_{31}$ in (9)-(11). There are two subcases in Case 1:

Case 1.1: $T_{12}^{d}(x) \in T_{12}^{*}(x)\left(Z_{12}=1\right)$, Case 1.2: $T_{12}^{d}(x) \notin T_{12}^{*}(x)\left(Z_{12}=0\right)$.

Case 1.1. We have $\operatorname{Pr}\left(\right.$ Case 1.1) $=\operatorname{Pr}\left(Z_{12}=Z_{23}=\right.$ $\left.Z_{31}=1\right)+\delta \operatorname{Pr}\left(Z_{12}=1, Z_{23}=Z_{31}=0\right)$, where $Z_{12}=Z_{23}=Z_{31}=1$ means all translators give correct translations and $Z_{12}=1, Z_{23}=Z_{31}=0$ means $T_{12}^{d}$ translates correctly but $T_{23}^{d}$ and $T_{31}^{d}$ both give incorrect translations. Only a small fraction happen to give correct translations at $S_{1}$, captured by $\delta$. By (13), $\operatorname{Pr}\left(Z_{12}=\right.$ $\left.Z_{23}=Z_{31}=1\right)=q_{12} q_{23} q_{31}+\lambda_{2}$. Now we compute $\operatorname{Pr}\left(Z_{12}=1, Z_{23}=Z_{31}=0\right)$.

$$
\begin{aligned}
& \operatorname{Pr}\left(Z_{12}=1, Z_{23}=Z_{31}=0\right) \\
= & \operatorname{Pr}\left(Z_{12}=1, Z_{23}=0\right)-\operatorname{Pr}\left(Z_{12}=1, Z_{23}=0, Z_{31}=1\right) \\
= & \operatorname{Pr}\left(Z_{12}=1, Z_{23}=0\right)-\operatorname{Pr}\left(Z_{12}=1, Z_{31}=1\right) \\
& +\operatorname{Pr}\left(Z_{12}=Z_{23}=Z_{31}=1\right) \\
= & q_{12}-\left(q_{12} q_{23}+\lambda_{1}\right)-\left(q_{12} q_{31}+\lambda_{1}\right)+q_{12} q_{23} q_{31}+\lambda_{2} \\
= & q_{12}\left(1-q_{23}\right)\left(1-q_{31}\right)+\lambda_{2}
\end{aligned}
$$

where the third equality is obtained by (12) and (13). So we have

$\operatorname{Pr}($ Case 1.1$)=q_{12} q_{23} q_{31}+\lambda_{2}+\delta\left(q_{12}\left(1-q_{23}\right)\left(1-q_{31}\right)+\lambda_{2}\right)$

Case 1.2. This case is possible only if $Z_{12}=0$, $Z_{23}+Z_{31} \leq 1$, which means $T_{12}^{d}$ gives incorrect 
translations; $T_{23}^{d}$ and $T_{31}^{d}$ do not give correct translations simultaneously. We write the probability of this case as $\operatorname{Pr}($ Case 1.2 $)=\delta\left(\operatorname{Pr}\left(Z_{12}=Z_{23}=Z_{31}=\right.\right.$ $0)+\operatorname{Pr}\left(Z_{12}=Z_{23}=0, Z_{31}=1\right)+\operatorname{Pr}\left(Z_{12}=0, Z_{23}=\right.$ $\left.\left.1, Z_{31}=0\right)\right)$.

To compute it, we have

$$
\begin{aligned}
& \operatorname{Pr}\left(Z_{12}=0, Z_{23}=1, Z_{31}=0\right) \\
= & \operatorname{Pr}\left(Z_{12}=0, Z_{23}=1\right)-\operatorname{Pr}\left(Z_{12}=0, Z_{23}=1, Z_{31}=1\right) \\
= & \operatorname{Pr}\left(Z_{23}=1\right)-\operatorname{Pr}\left(Z_{12}=Z_{23}=1\right)-\operatorname{Pr}\left(Z_{23}=Z_{31}=1\right) \\
& +\operatorname{Pr}\left(Z_{12}=1, Z_{23}=1, Z_{31}=1\right) \\
= & q_{23}-\left(q_{12} q_{23}+\lambda_{1}\right)-\left(q_{23} q_{31}+\lambda_{1}\right)+q_{12} q_{23} q_{31}+\lambda_{2} \\
= & \left(1-q_{12}\right) q_{23}\left(1-q_{31}\right)-2 \lambda_{1}+\lambda_{2}
\end{aligned}
$$

Similarly we can compute $\operatorname{Pr}\left(Z_{12}=Z_{23}=0, Z_{31}=\right.$ $1)=\left(1-q_{12}\right)\left(1-q_{23}\right) q_{31}-2 \lambda_{1}+\lambda_{2}$ and $\operatorname{Pr}\left(Z_{12}=\right.$ $\left.Z_{23}=Z_{31}=0\right)=\left(1-q_{12}\right)\left(1-q_{23}\right)\left(1-q_{31}\right)+3 \lambda_{1}-\lambda_{2}$. Then the probability of Case 1.2 is

$$
\operatorname{Pr}\left(\text { Case 1.2) }=\delta\left(1-q_{12}\right)\left(1-q_{23} q_{31}-\lambda_{1}+\lambda_{2}\right)\right.
$$

Case 2. The probability of Case 2 is simply the complement of Case 1.

$$
\operatorname{Pr}(\text { Case } 2)=1-\operatorname{Pr}(\text { Case } 1.1)-\operatorname{Pr}(\text { Case } 1.2)
$$

Then the accuracy of this triple learning is

$$
\begin{aligned}
q_{12}^{m} & =\operatorname{Pr}(\text { Case } 1.1)+\alpha^{\prime} \operatorname{Pr}(\text { Case } 2) \\
& =\left(1-\alpha^{\prime}\right) \operatorname{Pr}(\text { Case } 1.1)+\alpha^{\prime}(1-\operatorname{Pr}(\text { Case 1.2 }))
\end{aligned}
$$

where $\alpha^{\prime}$ is defined in (14). (15) is obtained by substitute (16) and (17) into (18).

Roles of $\lambda_{1}$ and $\lambda_{2}$. It is easy to see $q_{12}^{m}$ improves when $\lambda_{1}$ increases. For the impact of $\lambda_{2}$, we reorganize the term with $\lambda_{2}$ and have $(1+\delta) \lambda_{2}-\alpha^{\prime}(1+\delta) \lambda_{2}-\alpha^{\prime} \delta\left(1-q_{12}\right) \lambda_{2}$. $\alpha^{\prime}$ is generally not close to 1 , so a larger $\lambda_{2}$ helps in most cases. In the rest of our analysis we assume $\lambda_{1}=\lambda_{2}=0$ for simplification.

A similar hypothesis. Similar to the analysis of dual learning, we consider the condition where

$$
\frac{\alpha^{\prime}}{\beta^{\prime}}=\frac{\operatorname{Pr}\left(T_{12}^{d}(x) \in T_{12}^{*}(x), T_{31}^{d}\left(T_{23}^{d}\left(T_{12}^{d}(x)\right)\right) \in C(x)\right)}{\operatorname{Pr}\left(T_{12}^{d}(x) \notin T_{12}^{*}(x), T_{31}^{d}\left(T_{23}^{d}\left(T_{12}^{d}(x)\right)\right) \in C(x)\right)}
$$

and define $\Gamma^{\prime}=\gamma^{\prime} \operatorname{Pr}\left(T_{31}^{d}\left(T_{23}^{d}\left(T_{12}^{d}(x)\right)\right) \notin C(x)\right)$. Then the accuracy simplifies to

$$
\begin{aligned}
q_{12}^{m} & =\frac{\alpha^{\prime}\left(1-\Gamma^{\prime}\right)}{\alpha^{\prime}+\beta^{\prime}} \\
& =\frac{1-\Gamma^{\prime}}{1+\frac{\delta\left(1-q_{12}\right)\left(1-q_{23} q_{31}\right.}{q_{12} q_{23} q_{31}+\delta q_{12}\left(1-q_{23}\right)\left(1-q_{31}\right)}} \\
& =\frac{1-\Gamma^{\prime}}{1+M \frac{1-q_{12}}{q_{12}}},
\end{aligned}
$$

where $M=\frac{\delta\left(1-q_{23} q_{31}\right)}{q_{23} q_{31}+\delta\left(1-q_{23}\right)\left(1-q_{31}\right)}$. We observe that When $\gamma^{\prime}=0$ (and therefore $\Gamma^{\prime}=0$ ) and $M=1$, it simplifies to $q_{12}$, which is the accuracy of dual learning and that $q_{12}^{m}$ increases as $M$ decreases. To characterize the condition when $q_{12}^{m}>q_{12}$, we let $M<1$. We have $\frac{\delta\left(1-q_{23} q_{31}\right)}{q_{23} q_{31}+\delta\left(1-q_{23}\right)\left(1-q_{31}\right)}<1$, which leads to $q_{23}\left(\frac{2 \delta+1}{2 \delta} q_{31}-1\right)+q_{31}\left(\frac{2 \delta+1}{2 \delta} q_{23}-1\right)>0$. When $q_{23}, q_{31}>\frac{\delta}{\delta+0.5}$, which is also mild, multi-step dual learning outperforms dual learning.

\section{EXPERIMENTS OF DUAL LEARNING}

Since previous works [He et al. 2016, Xia et al. 2017a b. Wang et al., 2018, Xia et al. 2018] have demonstrated the strength of dual learning, we aim at providing some theoretical insights. We choose WMT14 English $\leftrightarrow$ Germen translation 1 ] and MultiUN [Eisele and Chen, 2010] English $\leftrightarrow$ French translation ${ }^{2}$ to verify our theoretical analysis for dual learning. For ease of reference, denote English, French, and German as En, Fr, and De respectively.

Datasets. Following the common practice in NMT, for the En $\leftrightarrow$ De tasks, we preprocess the data in the same way as that used in Ott et al. [2018], including tokenizing the words and applying BPE [Sennrich et al. 2016b] with $32 k$ merge operations. Eventually, we obtain $4.5 \mathrm{M}$ training sentence pairs. We concatenate newstest2012 and newstest 2013 as the validation set ( $6 K$ sentence pairs) and choose newstest 2014 as the test set ( $3 K$ sentence pairs). For the MultiUN En $\leftrightarrow$ Fr translation, following Ren et al. [2018], we sample $2 M / 6 K / 3 K$ sentence pairs as the training/validation/test sets. All sentences from MultiUN datasets are split into wordpiece following [Johnson et al. 2016]. To leverage dual learning, for WMT'14 En $\leftrightarrow$ De translation, we choose $40 M$ monolingual English sentences and $40 M$ monolingual German sentences from newscraw 3 . For MultiUN En $\leftrightarrow$ Fr translation, we randomly sample $1 M$ English and $1 M$ French sentences as the monolingual data to construct the duality loss.

Architecture. We use the Transformer model [Vaswani et al. 2017] for each translation task. For WMT En $\leftrightarrow$ De translation, we choose the transformer_big configuration, in which the word embedding dimension, hidden dimension and number of heads in multi-head attention are 1024, 4096 and 16 respectively. For MultiUN En $\leftrightarrow \mathrm{Fr}$ translation, we choose the transformer_base configuration, in which the aforementioned three numbers are 512 ,

\footnotetext{
${ }^{1}$ Data available at http://www.statmt.org/ wmt 14/translation-task.html

2 http://opus.nlpl.eu/Multiun.php 3 http://data.statmt.org/news-crawl/
} 
2048 and 8 respectively. Both transformer_big and transformer_base represent networks with six layers.

Optimization. We choose Adam [Kingma and Ba 2015] with inverse_sqrt learning rate scheduler [Vaswani] et al. 2017] to optimize the models. All experiments are conducted on eight GPUs. For WMT En $\leftrightarrow$ De tasks, following [Ott et al., 2018], we set the learning rate as $5 \times 10^{-4}$ and the batch size as 4096 tokens per GPU. The gradient is accumulated 16 times before update. For MultiUN tasks, the learning rate is $2 \times 10^{-4}$ and the batch size is 7168 tokens per GPU. All the models are trained until convergence.

Evaluation. We use beam search with beam width 4 to generate candidates. The evaluation metric is BLEU score [Papineni et al. 2002], which is a geometric mean of $n$-gram precisions $(n=1,2,3,4)$. We choose the script multi-bleu per $1^{4}$ to calculate BLEU scores. A large BLEU score indicates a better translation quality.

Translation qualities. The BLEU scores of all translation tasks are summarized in Table 2, in which the second row and third row represent the results of the standard Transformer and dual learning. We can see that after applying dual learning, the performances of all tasks are boosted. Specifically, on $\mathrm{En} \rightarrow \mathrm{De}$ and $\mathrm{De} \rightarrow$ En translation tasks, we can boost the baseline from 29.79 to 32.18 (2.39 points improvement), and from 34.15 to 38.06 (3.91 points improvement). On the other task, dual learning can achieve 0.65 and 0.86 point improvement, which demonstrates its effectiveness. We found that on MultiUN, we do not achieve as much improvement as WMT. The reason is that the MultiUN dataset is a collection of translated documents from the United Nations, which are usually of formal and simple patterns that are easy to learn. As a result, introducing more data might not increase the BLEU so much.

Table 2: BLEU scores of WMT2014 En $\leftrightarrow$ De and MultiUN En $\leftrightarrow$ Fr translations tasks.

\begin{tabular}{ccccc}
\hline & En $\rightarrow$ De & De $\rightarrow$ En & En $\rightarrow$ Fr & $\mathrm{Fr} \rightarrow$ En \\
\hline Vanilla & 29.79 & 34.15 & 50.26 & 50.56 \\
Dual & 32.18 & 38.06 & 50.91 & 51.42 \\
\hline
\end{tabular}

We are aware that back translation [Sennrich et al. 2016a] is another baseline of leveraging monolingual data. We apply this technique to WMT En $\rightarrow$ De and De $\rightarrow$ En. We obtain 30.43 and 37.17 BLEU scores respectively, which are not as good as dual learning. We leave the study of back translation as future work.

${ }^{4}$ https://github.com/moses-smt/ mosesdecoder/blob/master/scripts/generic/ multi-bleu.perl
Table 3: Accuracy of Translators Using Different Threshold BLEU.

\begin{tabular}{cccc}
\hline \multicolumn{2}{c}{ Threshold BLEU } & 10 & 20 \\
\hline \multirow{4}{*}{ En $\leftrightarrow$ De } & $p_{12}$ & 0.65 & 0.54 \\
& $p_{21}$ & 0.73 & 0.65 \\
& $p_{12}^{d}$ & 0.70 & 0.60 \\
& $p_{21}^{d}$ & 0.77 & 0.70 \\
\hline \multirow{4}{*}{ En $\leftrightarrow$ Fr } & $p_{12}$ & 0.82 & 0.77 \\
& $p_{21}$ & 0.80 & 0.74 \\
& $p_{12}^{d}$ & 0.82 & 0.78 \\
& $p_{21}^{d}$ & 0.81 & 0.75 \\
\hline
\end{tabular}

Translator accuracy. We interpret the results in terms of accuracy, i.e., the $p_{i j}$ and $p_{i j}^{d}$ in Table 1. A sentence is regarded to be correctly translated if the corresponding BLEU score is larger than a given threshold BLEU score. We choose threshold BLEU score to be 10 and 20 .

The accuracy of each translator is shown in Table 3 . Let $S_{1}$ and $S_{2}$ denote English and German respectively for the En $\leftrightarrow$ De task (English and French respectively for the En $\leftrightarrow$ Fr task). Values are percentages of translations that are above the threshold. Qualitatively, we observe that dual learning outcomes are better than standard transformers. More interesting observations lie in the following quantitative analysis on $\mathrm{En} \leftrightarrow$ De task.

Evaluation of Assumption 1 and empirical $\alpha, \beta, \gamma$. For a given test dataset, we define the empirical estimate of $\alpha$, $\beta$, and $\gamma$ as follows.

$$
\begin{aligned}
& \hat{\alpha}=\frac{\# \text { of } x \mid T_{12}^{d}(x) \in T_{12}^{*}(x), T_{21}^{d}\left(T_{12}^{d}(x)\right) \in C(x)}{\# \text { of } x \mid T_{21}\left(T_{12}(x)\right) \notin C(x)} \\
& \hat{\beta}=\frac{\# \text { of } x \mid T_{12}^{d}(x) \notin T_{12}^{*}(x), T_{21}^{d}\left(T_{12}^{d}(x)\right) \in C(x)}{\# \text { of } x \mid T_{21}\left(T_{12}(x)\right) \notin C(x)} \\
& \hat{\gamma}=\frac{\# \text { of } x \mid T_{21}^{d}\left(T_{12}^{d}(x)\right) \notin C(x)}{\# \text { of } x \mid T_{21}\left(T_{12}(x)\right) \notin C(x)}
\end{aligned}
$$

To evaluate Assumption 1 , we define

$$
\eta=\frac{\# \text { of } x \mid T_{21}^{d}\left(T_{12}^{d}(x)\right) \in C(x)}{\# \text { of } x \mid T_{21}\left(T_{12}(x)\right) \in C(x)}
$$

Ideally $\eta=1$. The empirical estimates using the $\mathrm{En} \leftrightarrow \mathrm{De}$ test data under threshold BLEU scores 10 and 20 are shown in Table 4 In each cell, the values on the left are for $\mathrm{En} \rightarrow$ De direction and the values on the right are for $\mathrm{De} \rightarrow$ En direction. We observe that $\eta$ values are close to 1, which means Assumption 1 is reasonable. The high $\hat{\gamma}$ values indicate that the reconstruction loss is still high after dual learning. Therefore, we believe there exist approaches to improve dual learning and how to further reduce the reconstruction loss is a promising direction. 
Table 4: Estimated Parameters Using Test Data.

\begin{tabular}{ccc}
\hline Threshold BLEU & 10 & 20 \\
\hline$\eta$ & $96.8 \% \mid 94.3 \%$ & $96.4 \% \mid 93.3 \%$ \\
$\alpha$ & $0.30 \mid 0.31$ & $0.27 \mid 0.32$ \\
$\beta$ & $0.28 \mid 0.23$ & $0.32 \mid 0.24$ \\
$\gamma$ & $0.42 \mid 0.45$ & $0.41 \mid 0.44$ \\
\hline
\end{tabular}

\section{EXPERIMENTS OF MULTI-STEP DUAL LEARNING}

To verify the effectiveness of multi-step dual learning, we work on the translation between English (En), French (Fr) and Spanish (Es). Again, we choose to use the MultiUN dataset to train the translation models since any two of the aforementioned three languages have bilingual sentence pairs. We study two different settings, where for each language pair, we are provided with $2 M$ or $0.2 M$ bilingual sentence pairs. For both settings, we choose $1 M$ monolingual sentences for each language. We use transformer_base for all experiments in this section, where the model is a six-block network, with word embedding size, hidden dimension size and number of heads 512 , 2048 and 6 . The training process is the same as that in Section 5

Table 5: Experimental Results on MultiUN (2M bilingual data)

\begin{tabular}{lccc}
\hline & Vanilla & Dual & Multi-step \\
\hline $\mathrm{En} \rightarrow \mathrm{Fr}$ & 50.26 & 50.91 & 51.28 \\
$\mathrm{Fr} \rightarrow \mathrm{En}$ & 50.56 & 51.42 & 51.89 \\
$\mathrm{En} \rightarrow \mathrm{Es}$ & 55.15 & 55.51 & 55.97 \\
$\mathrm{Es} \rightarrow \mathrm{En}$ & 55.23 & 55.77 & 56.17 \\
$\mathrm{Es} \rightarrow \mathrm{Fr}$ & 47.75 & 48.23 & 48.62 \\
$\mathrm{Fr} \rightarrow \mathrm{Es}$ & 48.13 & 48.52 & 48.87 \\
\hline
\end{tabular}

The experimental results of using $2 M$ bilingual data and $1 M$ monolingual data are shown in Table 5. We can see that on average, dual learning can boost the six baselines (i.e., standard transformer) by 0.55 point. Although dual learning can achieve very high scores on MultiUN translation tasks, our proposed multi-step dual learning can still improve it by 0.41 point on average.

The results of using $0.2 \mathrm{M}$ bilingual data plus $1 \mathrm{M}$ monolingual data is shown in Table 6 We have the following observations:

(1) Since there are fewer bilingual sentences, the baselines of the six translation tasks are not as good as those in Table 5 .
(2) For this setting, dual learning can improve the BLEU scores by 1.93 points on average, which is consistent with the discovery in He et al. [2016] that dual learning can obtain more improvements when the number of bilingual sentences is small.

(3) When multi-step dual learning is added to the conventional dual learning, we can achieve extra 1.45 improvements on average, which demonstrates the effectiveness of multi-step dual learning. We also observe that multistep dual learning can bring more improvement when the number of labeled data is limited.

Table 6: Experimental Results on MultiUN (0.2M bilingual data)

\begin{tabular}{cccc}
\hline & Vanilla & Dual & Multi-step \\
\hline $\mathrm{En} \rightarrow \mathrm{Fr}$ & 43.12 & 45.54 & 47.23 \\
$\mathrm{Fr} \rightarrow \mathrm{En}$ & 43.26 & 45.44 & 46.72 \\
$\mathrm{En} \rightarrow \mathrm{Es}$ & 49.28 & 51.07 & 52.56 \\
$\mathrm{Es} \rightarrow \mathrm{En}$ & 47.80 & 50.31 & 51.65 \\
$\mathrm{Es} \rightarrow \mathrm{Fr}$ & 41.47 & 42.81 & 43.52 \\
$\mathrm{Fr} \rightarrow \mathrm{Es}$ & 41.21 & 42.57 & 44.97 \\
\hline
\end{tabular}

\section{CONCLUSIONS}

We provide the first theoretical study of dual learning and characterize conditions when dual learning outperforms vanilla translators. We also propose an algorithmic extension of dual learning, the multi-step dual learning framework, which is provably better than dual learning under mild conditions. Our dual learning experiments demonstrate the efficacy of dual learning w.r.t. accuracy and provide insights into the potential power of dual learning. Our experiments on multi-step dual learning framework show further improvement from dual learning.

\section{References}

Mikel Artetxe, Gorka Labaka, Eneko Agirre, and Kyunghyun Cho. Unsupervised neural machine translation. In International Conference on Learning Representations (ICLR 2018), 2018.

Yong Cheng, Wei Xu, Zhongjun He, Wei He, Hua Wu, Maosong Sun, and Yang Liu. Semi-supervised learning for neural machine translation. In $A C L$, volume 1, pages 1965-1974, 2016.

Sergey Edunov, Myle Ott, Michael Auli, and David Grangier. Understanding back-translation at scale. In Proceedings of the 2018 Conference on Empirical Methods in Natural Language Processing, pages 489500, 2018. 
Andreas Eisele and Yu Chen. Multiun: A multilingual corpus from united nation documents. In Proceedings of the Seventh conference on International Language Resources and Evaluation, pages 2868-2872, 52010.

Tomer Galanti, Lior Wolf, and Sagie Benaim. The role of minimal complexity functions in unsupervised learning of semantic mappings. In 6th International Conference on Learning Representations, 2018.

Di He, Yingce Xia, Tao Qin, Liwei Wang, Nenghai Yu, Tieyan Liu, and Wei-Ying Ma. Dual learning for machine translation. In Advances in Neural Information Processing Systems, pages 820-828, 2016.

Melvin Johnson, Mike Schuster, Quoc V Le, Maxim Krikun, Yonghui Wu, Zhifeng Chen, Nikhil Thorat, Fernanda Viégas, Martin Wattenberg, Greg Corrado, et al. Google's multilingual neural machine translation system: enabling zero-shot translation. arXiv preprint arXiv:1611.04558, 2016.

Taeksoo Kim, Moonsu Cha, Hyunsoo Kim, Jung Kwon Lee, and Jiwon Kim. Learning to discover crossdomain relations with generative adversarial networks. In International Conference on Machine Learning, pages 1857-1865, 2017.

Diederik P Kingma and Jimmy Ba. Adam: A method for stochastic optimization. ICLR, 2015.

Guillaume Lample, Alexis Conneau, Ludovic Denoyer, and Marc'Aurelio Ranzato. Unsupervised machine translation using monolingual corpora only. In International Conference on Learning Representations (ICLR 2018), 2018.

Ping Luo, Guangrun Wang, Liang Lin, and Xiaogang Wang. Deep dual learning for semantic image segmentation. In Proceedings of the IEEE Conference on Computer Vision and Pattern Recognition, Honolulu, HI, USA, pages 21-26, 2017.

Myle Ott, Sergey Edunov, David Grangier, and Michael Auli. Scaling neural machine translation. arXiv preprint arXiv:1806.00187, 2018.

Kishore Papineni, Salim Roukos, Todd Ward, and WeiJing Zhu. Bleu: a method for automatic evaluation of machine translation. In Proceedings of the 40th annual meeting on association for computational linguistics, pages 311-318. Association for Computational Linguistics, 2002.

Alberto Poncelas, Dimitar Shterionov, Andy Way, Gideon Maillette de Buy Wenniger, and Peyman Passban. Investigating backtranslation in neural machine translation. In Proceedings of the 21st Annual Conference of the European Association for Machine Translation: 28-30 May 2018, Universitat d'Alacant, Alacant,
Spain, pages 249-258. European Association for Machine Translation, 2018.

Shuo Ren, Wenhu Chen, Shujie Liu, Mu Li, Ming Zhou, and Shuai Ma. Triangular architecture for rare language translation. ACL, 2018.

Rico Sennrich, Barry Haddow, and Alexandra Birch. Improving neural machine translation models with monolingual data. In Proceedings of the 54th Annual Meeting of the Association for Computational Linguistics (Volume 1: Long Papers), pages 86-96, Berlin, Germany, August 2016a. Association for Computational Linguistics. doi: 10.18653/v1/P16-1009. URL https: //www.aclweb.org/anthology/P16-1009.

Rico Sennrich, Barry Haddow, and Alexandra Birch. Neural machine translation of rare words with subword units. $A C L, 2016 \mathrm{~b}$.

Duyu Tang, Nan Duan, Tao Qin, Zhao Yan, and Ming Zhou. Question answering and question generation as dual tasks. arXiv preprint arXiv:1706.02027, 2017.

Ashish Vaswani, Noam Shazeer, Niki Parmar, Jakob Uszkoreit, Llion Jones, Aidan N Gomez, Łukasz Kaiser, and Illia Polosukhin. Attention is all you need. In Advances in Neural Information Processing Systems, pages 5998-6008, 2017.

Yijun Wang, Yingce Xia, Li Zhao, Jiang Bian, Tao Qin, Guiquan Liu, and T Liu. Dual transfer learning for neural machine translation with marginal distribution regularization. In Proceedings of the Thirty-Second AAAI Conference on Artificial Intelligence, 2018.

Yingce Xia, Jiang Bian, Tao Qin, Nenghai Yu, and TieYan Liu. Dual inference for machine learning. In Proceedings of the Twenty-Sixth International Joint Conference on Artificial Intelligence, IJCAI-17, pages 3112-3118, 2017a.

Yingce Xia, Tao Qin, Wei Chen, Jiang Bian, Nenghai $\mathrm{Yu}$, and Tie-Yan Liu. Dual supervised learning. In International Conference on Machine Learning, pages 3789-3798, 2017b.

Yingce Xia, Xu Tan, Fei Tian, Tao Qin, Nenghai Yu, and Tie-Yan Liu. Model-level dual learning. In International Conference on Machine Learning, pages 3789-3798, 2018.

Jun-Yan Zhu, Taesung Park, Phillip Isola, and Alexei A Efros. Unpaired image-to-image translation using cycle-consistent adversarial networks. In IEEE International Conference on Computer Vision, 2017. 


\section{A Derivation}

We leverage the negative logarithmic probability to measure the differences between the original $x^{(2)}$ and the reconstructed one. Let $\mathcal{R}\left(x^{(2)}\right)$ denote the event that after passing the loop $S_{2} \rightarrow S_{k} \rightarrow S_{1} \rightarrow S_{2}, x^{(2)}$ is reconstructed to $x^{(2)}$. We have that

$$
\begin{aligned}
& \ln \operatorname{Pr}\left(\mathcal{R}\left(x^{(2)}\right)\right)=\sum_{x^{(k)} \in S_{k}} \sum_{x^{(1)} \in S_{1}} \ln \operatorname{Pr}\left(x^{(2)}, x^{(1)}, x^{(k)} \mid\right. \\
& \text { starting from } \left.x^{(2)}, \text { applied by } \theta_{2 k}, \theta_{k 1}, \theta_{12} \text { sequentially }\right) \\
= & \sum_{x^{(k)} \in S_{k}} \sum_{x^{(1)} \in S_{1}} \ln \operatorname{Pr}\left(x^{(1)}, x^{(k)} \mid x^{(2)} ; \theta_{2 k}, \theta_{k 1}\right) . \\
\geq & \sum_{x^{(k)} \in S_{k}} \sum_{x^{(1)} \in S_{1}} \operatorname{Pr}\left(x^{(1)}, x^{(k)} \mid x^{(2)} ; \theta_{2 k}, \theta_{k 1}\right) . \\
= & \sum_{x^{(k)} \in S_{k}} \sum_{x^{(1)} \in S_{1}} \operatorname{Pr}\left(x^{(1)}, x^{(k)} \mid x^{(2)} ; \theta_{2 k}, \theta_{k 1}\right) . \\
= & \sum_{x^{(k)} \in S_{k}} \sum_{x^{(1)} \in S_{1}} \operatorname{Pr}\left(x^{(k)} \mid x^{(2)} ; \theta_{2 k}\right) \operatorname{Pr}\left(x^{(1)} \mid x^{(k)} ; \theta_{k 1}\right) .
\end{aligned}
$$

$$
\ln \operatorname{Pr}\left(x^{(2)} \mid x^{(1)} ; \theta_{12}\right)
$$

$=\mathbb{E}_{x^{(k)} \sim \operatorname{Pr}\left(\cdot \mid x^{(2)} ; \theta_{2 k}\right)} \mathbb{E}_{x^{(1)} \sim \operatorname{Pr}\left(\cdot \mid x^{(k)} ; \theta_{k 1}\right)} \ln \operatorname{Pr}\left(x^{(2)} \mid x^{(1)} ; \theta_{12}\right)$.

In Eqn.(19), the first Pr represents the jointly probability that $x^{(2)}$ can be translated into $x^{(k)}$ with $\theta_{2 k}$, and the the obtained $x^{(k)}$ can be translated into $x^{(1)}$ with $\theta_{k 1}$; the second $\operatorname{Pr}$ represents the probability that given $x^{(1)}$, it can be translated back to $x^{(2)}$ with $\theta_{12}$. 